\title{
Question-answer dynamics and confirmation theory in reasoning by representativeness
}

\author{
Mathias Sablé-Meyer ${ }^{1}$ \\ Janek Guerrini² \\ Salvador Mascarenhas ${ }^{2}$ \\ ${ }^{1}$ UNICOG; CEA; INSERM; Université Paris-Saclay; NeuroSpin; Saclay France \\ Collège de France; Paris; France \\ 2Institut Jean-Nicod; Département d'études cognitives \\ ENS; EHESS; PSL University; Paris France; CNRS
}

\begin{abstract}
We show that probabilistic decision-making behavior characteristic of reasoning by representativeness or typicality arises in minimalistic settings lacking many of the features previously thought to be necessary conditions for the phenomenon. Specifically, we develop a version of a classical experiment by Kahneman and Tversky (1973) on base-rate neglect, where participants have full access to the probabilistic distribution, conveyed entirely visually and without reliance on familiar stereotypes, rich descriptions, or individuating information. We argue that the notion of evidential support as studied in (Bayesian) confirmation theory offers a good account of our experimental findings, as has been proposed for related data points from the representativeness literature. In a nutshell, when faced with competing alternatives to choose from, humans are sometimes less interested in picking the option with the highest probability of being true (posterior probability), and instead choose the option best supported by available evidence. We point out that this theoretical avenue is descriptively powerful, but has an as-yet unclear explanatory dimension. Building on approaches to reasoning from linguistic semantics, we propose that the chief trigger of confirmation-theoretic mechanisms in deliberate reasoning is a linguistically-motivated tendency to interpret certain experimental setups as intrinsically contrastive, in a way best cashed out by modern linguistic semantic theories of questions. These questions generate pragmatic pressures for interpreting surrounding information as having been meant to help answer the question, which will naturally give rise to confirmation-theoretic effects, very plausibly as a byproduct of iterated Bayesian update as proposed by modern Bayesian theories of relevance-based reasoning in pragmatics. Our experiment provides preliminary but tantalizing evidence in favor of this hypothesis, as participants displayed significantly more confirmation-theoretic behavior in a condition that highlighted the question-like, contrastive nature of the task.
\end{abstract}

Keywords: reasoning; decision making; base-rate neglect; question-answer dynamics

\section{Introduction}

Reasoning tasks often lead to apparent systematic deviations from rationality in humans, which can in principle illuminate the functional aim of the human faculty of reasoning, as well as the processes that underlie it. But this line of inquiry is only as solid as the extent to which we can be confident that the observed apparent deviation from rationality is more than just an artifact of the experimental design.

The lawyers-and-engineers experimental paradigm from the heuristics and biases literature illustrates this tension very clearly. In the classical study by Kahneman and Tversky (1973), participants were given prior probabilities on the distribution of two professions in a collection of one-hundred individuals: lawyers and engineers. They were told that psy- chologists had composed personality descriptions of each of these individuals. Then they saw the personality description of a randomly selected individual from this pool, and they were tasked with guessing the probability that this individual was an engineer. The descriptions were linguistically rich, provided abundant individuating information, and highlighted traits stereotypically associated with one or the other profession. For example, "Jack is a 45-year-old man. He is married and has four children. He is generally conservative, careful, and ambitious. He shows no interest in political and social issues and spends most of his free time on his many hobbies, which include home carpentry, sailing, and mathematical puzzles." Participants' responses were entirely determined by the content of these personality descriptions, displaying no variation between conditions that substantively 
shifted the prior probabilities on the professions between participants. Kahneman and Tversky (1973) concluded that, in the presence of individuating information and rich stereotypes, humans eschew prior probabilities and make decisions based purely on typicality judgments.

Later research cast serious doubt on this conclusion. Most notably, Gigerenzer and Hoffrage (1995) have shown that framing these kinds of tasks in terms of frequencies (3 out of 50) rather than probabilities $(6 \%)$ makes the irrational responses all but disappear. This and related results demonstrate that the base-rate neglect bias identified by Kahneman and Tversky (1973), if real, is highly dependent on features of the experimental task. Accordingly, sweeping conclusions about humans' inability to reason normatively with probabilities are unwarranted.

Yet, participants' behavior in the versions of the lawyersand-engineers task that $d o$ display irrationality is nevertheless in need of an explanation. Humans do not overall neglect prior probabilities, but in those conditions where they do, what mechanism produces their non-normative response, and what concrete aspects of the task prompt them to deploy this mechanism?

\section{Representativeness errors and confirmation theory}

In Kahneman and Tversky's view, base-rate neglect as illustrated by the original lawyers-and-engineers task was just one example of the failures of reasoning brought about by a human tendency to deploy reasoning by typicality (representativeness) in contexts where the norm would not seem to call for it. Another example was the conjunction fallacy (Tversky \& Kahneman, 1983). In the best-known version of this paradigm, participants first see a rich description of an individual named Linda, stating that she participated in progressive activist movements in her youth, and must then pick one of two options as the most probable. One of the options ("bank teller active in the feminist movement") is the conjunction of the other option ("bank teller") with a property intuitively quite closely related to the description of the individual as a matter of typicality reasoning ("active in the feminist movement"). Participants overwhelmingly chose the conjunction as the most probable option, in apparent violation of the classical probability calculus. This conjunction fallacy has inspired a host of theories offering alternatives to the original account in terms of representativeness.

One particularly successful view attributes this conjunction mistake to confirmation-theoretic reasoning (Crupi et al., 2008; Tentori et al., 2013; see also Tenenbaum \& Griffiths, 2001, on other aspects of reasoning by representativeness). Participants in conjunction fallacy tasks engage in an evidentiary process, where they consider the two options ("bank teller" and "bank teller active in the feminist movement") as competing hypotheses about Linda, and the rich description as evidence adduced to decide between the two hypotheses.
Informally, a "bank teller active in the feminist movement" theory is far better supported by the available evidence (the description of Linda) than the alternative theory "bank teller." This general account can be cashed out by appeal to any of a number of Bayesian measures of evidential support (Crupi et al., 2008). A particularly perspicuous one is the Difference measure, which, for a hypothesis $h$ and piece of evidence $e$, defines the extent to which $e$ supports $h$ as

$$
D(h, e)=P(h \mid e)-P(h),
$$

that is the posterior probability of the hypothesis minus the prior. Intuitively, this is a measure of the extent to which learning the evidence increases one's belief in the hypothesis.

We propose that this theoretical approach transfers to the lawyers-and-engineers paradigm. If reasoners are engaging in the same kind of evidentiary process, that means they are asking themselves whether the evidence given (the description of Jack) better supports a "lawyer" hypothesis or an "engineer" hypothesis. Using the same Difference measure as above, it is clear that the probability on "lawyer" does not increase relative to its prior upon learning the facts in the description, while that of "engineer" does increase, relative to its prior. In this way of conceiving of the task, disregarding prior probabilities is well understood, since what matters for the Difference measure is the increase in credence, rather than the starting point.

\section{Questions and alternatives in reasoning}

Under what conditions do humans deploy this confirmation-theoretic mechanism, and why? We propose that, rather than being an outright mistake revealing deep-seated human irrationality, this kind of behavior is a perfectly rational response to structural cues and thinly-veiled pragmatic pressures of the relevant experimental designs, urging participants to maximize the utility of the evidence given as a means of choosing between alternative possibilities.

Sablé-Meyer and Mascarenhas (2021) show that confirmation theory offers an account of seemingly unrelated problems from ostensibly deductive reasoning. The following is a representative example of their experimental materials.

The gun fired and the guitar was out of tune, or someone was hiding in the attic.

The trigger was pulled.

Does it follow that the guitar was out of tune?

This inference was overwhelmingly accepted by participants $(71.8 \pm 5.7 \%)$. Notice that, while it feels like a sensible probabilistic inference from ordinary reasoning, it is actually not at all clear in what way it would conform to the standard norms of Bayesian rationality in naive human reasoning, namely posterior probabilities (Oaksford \& Chater, 
2007). In particular, even if the probability of "the gun fired" conditional on "the trigger was pulled" were 1 , there is no rational guarantee that the probability of "the guitar was out of tune" will be higher than that of "someone was hiding in the attic." Yet, humans presented with a problem like this will reliably conclude that "the guitar was out of tune," never that "someone was hiding in the attic."

Sablé-Meyer and Mascarenhas (2021) argue that the right explanation for this kind of reasoning problem involves elements from linguistic semantics and from confirmation theory. Following the account of question-answer dynamics in reasoning by Koralus and Mascarenhas (2013), Sablé-Meyer and Mascarenhas (2021) propose that the first premise, by virtue of being a disjunction (expressed by the connective "or" in this example), presents a question. This move is motivated by a host of results from linguistics connecting the morphology and the semantics of disjunctions with those of interrogatives, across unrelated language families (AlonsoOvalle, 2006; Groenendijk, 2008; Jayaseelan, 2001, 2008; Kratzer \& Shimoyama, 2002; Mascarenhas, 2009; Szabolcsi et al., 2014). In this view, a reasoner who has interpreted the first premise is now entertaining the following question: is it that the gun fired and the guitar was out of tune, or is it that someone was hiding in the attic? The reasoner will then naturally attempt to interpret other information in the problem as an answer to that question. The second premise "the trigger was pulled" offers just that: interpreted as an answer meant to help choose one of the two alternative possibilities under consideration, elementary criteria of pragmatic relevance tell us it will be interpreted to suggest that "the gun fired and the guitar was out of tune," rather than its competing alternative "someone was hiding in the attic."

This kind of relevance reasoning can be naturally cashed out in terms of confirmation theory. The answer (second premise) is meant to help me decide between two possibilities, so I will check which of these two possibilities is the information in the answer better evidence for. Following the Difference measure, it is clear that learning that "the trigger was pulled" will raise the probability of "the gun fired and the guitar was out of tune" to a far greater extent than it will raise the probability of "someone was hiding in the attic." Other confirmation measures than Difference yield equivalent results in a binary decision task such as this.

Moreover, the idea that confirmation theory plays a role in relevance considerations is a key byproduct of one of the most successful theories of pragmatics in recent years, the Rational Speech Act model (Frank \& Goodman, 2012; Goodman \& Frank, 2016). In the RSA, the meaning of utterances is constructed from their literal meaning and iterated Bayesian update, considering the knowledge of the speaker, the listener, and the context. An interpretation with a high posterior probability can be dispreferred in favor of an interpretation with a lower posterior, effectively promoting the initial likelihood of the interpretation over its posterior probability.

Lastly, there is already some evidence that pragmatic pressures were crucially at play in Kahneman and Tversky's (1973) original experiment. (Schwarz et al., 1991) manipulated what we would call the answer component of the problem (the description of Jack), as opposed to the question component which is of chief interest to us here. They conducted a study where the information about the individual randomly selected was relayed to participants by a computer, significantly mitigating mistakes and promoting posteriorprobability reasoning. This manipulation discourages participants from thinking that the description is being presented expressly as information meant to be used to decide between the possible alternatives presented by the question, by using an agent (the computer in 1991) far less likely to conform to human standards of communication. In our terms then, Schwarz et al. (1991) successfully blocked question-answer dynamics by interfering with the default presumed speaker intentions behind the description.

With this explanation for the problem at hand and structurally similar ones, Sablé-Meyer and Mascarenhas (2021) unify confirmation-theoretic accounts of problems from the representativeness literature, such as the conjunction fallacy, with ostensibly deductive problems, extending the descriptive and explanatory breadth of confirmation-theoretic accounts of reasoning.

We propose that this explanation extends to the lawyersand-engineers paradigm. If this hypothesis is right, then we have the following three expectations.

1. Contra Kahneman and Tversky (1973), the phenomenon should not depend on familiar stereotypes and individuating information provided by means of rich descriptions.

2. It should not depend on language proper, but on communication, that is to say, it should be possible to prompt the same behavior with non-linguistic materials, as long as the communicative nature of the situation is not lost, in order to allow for pragmatic processes to kick in.

3. The phenomenon should depend heavily on structural cues that highlight the two possible alternatives; in other words, the degree to which the question element is salient should have a reflection on the extent to which reasoners engage in confirmation-theoretic reasoning.

This article offers an experimental investigation of this hypothesis for the lawyers-and-engineers paradigm. We aimed to show not just that confirmation-theoretic reasoning offers an account of deviations from the norm when they occur in the lawyers-and-engineers paradigm, but also that this happens even in the absence of stereotypes and linguistically rich 
descriptions, elements deemed critical in representativeness accounts, and in the presence of complete information about the relevant probability distribution, with minimal use of language, thereby eschewing both probability and frequency talk. Additionally, we set out to test the prediction that the salience of the question element played a key role in the phenomenon.

\section{Lawyers and engineers, distilled}

In order to remove stereotypes and linguistically rich descriptions from the equation, an experimental design has to eschew real-life scenarios and use entirely ad hoc situations instead. Additionally, the probabilistic distribution should be fully accessible to the participants at an immediate intuitive level. Specifically, the distribution should not be conveyed by means of mathematical language, frequentist or probabilistic, lest we increase the chances of unintended interpretations by participants. What's more, conveying probability distributions linguistically introduces the danger of priming participants to pay particular attention to certain elements of the distribution, for example by explicitly describing the prior probabilities and relevant conditional probabilities. In our study, we conveyed distributions purely visually, but in principle other modalities would be appropriate as well.

To avoid reliance on individuating information is much simpler: we need only make the elements of the distribution vary exclusively with respect to the two dimensions that are central to the design. That is, one needs two categories (lawyers and engineers), which in the simplest case ought to be mutually exclusive, and a piece of information that can be true or false of each of the members of these categories ("shows no interest in political issues").

However, conveying the distribution without language also removes potentially crucial attentional cues. In particular, it could impact the salience of the question element of the problem.

Confirmation-theoretic reasoning depends on a contrastive framing of the problem at hand, in Sablé-Meyer and Mascarenhas's (2021) view. For the case of lawyers and engineers, the prediction would be that fewer mistakes will occur if participants do not conceive of the task as being primarily about choosing between lawyers and engineers. Given that the classical, language-based versions of the task pit lawyers against engineers, explicitly describing their prior distributions in each trial, the linguistic setup is plausibly inextricably contrastive in the relevant sense. But a visual counterpart of this paradigm isn't necessarily bound to the same framing. Indeed, it is in principle possible to visually convey a probability distribution involving two categories without explicitly drawing attention to those two categories. Our study tests for this hypothesis by using two kinds of visual framing, one making the question element of the problem salient, thereby encouraging contrastive reasoning re- garding the two categories, the other discouraging it.

\section{Methods}

The task in our experiment was to choose between two shapes (our hypotheses) as a function of information about their color (the evidence). In each trial, participants saw a fresh distribution of 100 elements, each falling into one of two shapes and one of two colors. They then had to guess the shape of an element knowing only its color, and the fact that it had been randomly sampled from the distribution on screen. We give an example of a trial in Figure 1.A.

We were interested in participants' rationality as a function of the terms of Bayes' theorem. The norm for rationality is to respond entirely as a function of the probabilities of each shape conditional on the observed color (posteriors). Yet, we hypothesized that confirmation-theoretic measures, like Difference as discussed above, would interfere with a rational strategy. In our minimalistic binary design, all confirmationtheoretic measures make identical predictions, which align with the likelihood term of Bayes' theorem, that is the probability that an element is of a given color, conditional on its being of a certain shape (proof in supplementary materials).

Our design disentangles posteriors and likelihoods by employing both congruent trials, where posteriors and likelihoods make the same prediction, and incongruent trials, where they diverge. This factor was tested within subjects. More generally, we conjectured that the difference in likelihoods between the normatively correct hypothesis and the alternative hypothesis $\left(\Delta_{\text {likelihood }}\right)$ would drive the salience of the confirmation-theoretic strategy, thereby pulling participants away from normatively rational behavior. In other words, extreme differences between the likelihoods of the two hypotheses should promote the non-normative strategy. Conversely, extreme posterior probabilities should promote the normative strategy. For consistency, we operationalized the salience of posteriors as a difference between the posterior of the normatively correct option and the posterior of its competitor, which we write $\Delta_{\text {posteriors }}$ Knowing one posterior is enough to reconstruct the other, but using the difference ensures that (i) $\Delta_{\text {posteriors }}>0$ as we remove the lower posterior from the higher one, and that (ii) the sign of $\Delta_{\text {likelihoods }}$ aligns with congruency: a positive sign indicates that posteriors and likelihoods predict the same answer, while a negative sign indicates that they differ.

Recall that, based on theoretical work on the conjunction fallacy and deductive reasoning, we further hypothesized that the comparative nature of the task matters greatly: saliently contrasting the two possible answers in the visual setup should highlight the question-like nature of this setup, inducing pragmatic mechanisms and confirmation-theoretic behavior more strongly. Our experiment had two conditions meant to test this hypothesis. In what we call the flat condition, all elements were displayed in a single box, ordered by 


\section{Figure 1}

\section{Example and structure of our task}

(A) Example of a trial, including the response modality. $(\boldsymbol{B}, \boldsymbol{C})$ Examples of conditions in our task. In all four examples, the evidence is "the sampled object happened to be green," and the normatively rational answer is "square." In congruent trials (left columns) posteriors and likelihoods coincide, while in incongruent trials they do not. Participants were either in the "flat" condition $(B)$ or in the "structured" condition $(C)$. (D) Examples of monocolor control trials. Any participant that answered "equally likely" was removed from the analysis.

A. Example of a trial

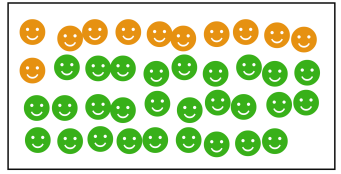
It happens to be a green object. Which of the following would you guess it is?

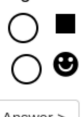

shape and color. The structured condition showed elements in two side-by-side boxes according to their shape, internally ordered by color. This was a between-subjects factor.

In Figure 1.B and Figure 1.C we show examples of trials in each cell of the $2 \times 2$ design. On the figures, the hypotheses are the two shapes, squares and smilies, and the evidence is the color green. In congruent trials (left) in both flat and structured condition, green squares are more numerous than green smilies (higher posterior for squares). At the same time, the proportion of squares that are green is greater than the proportion of smilies that are green (higher likelihood). By contrast, incongruent trials pit posteriors against likelihoods: there are still more green squares than green smilies (posteriors), but now the proportion of smilies that are green is greater than the proportion of squares that are green (likelihoods).

Participants. We recruited $N=105$ participants for an online experiment conducted on Amazon's Mechanical Turk crowdsourcing platform. Fifty-three in the flat condition and 52 in the structured condition (58 females, 45 males, two preferred not to answer; age distribution: 18-24: 10; 25-34: 29; 35-44: 26; 45-54: 20; 55-64: 10; 65-74: 8; two preferred not to answer.) All participants were compensated for their participation.

Procedure. The experiment was programmed in jsPsych (De Leeuw, 2015), with custom libraries built in

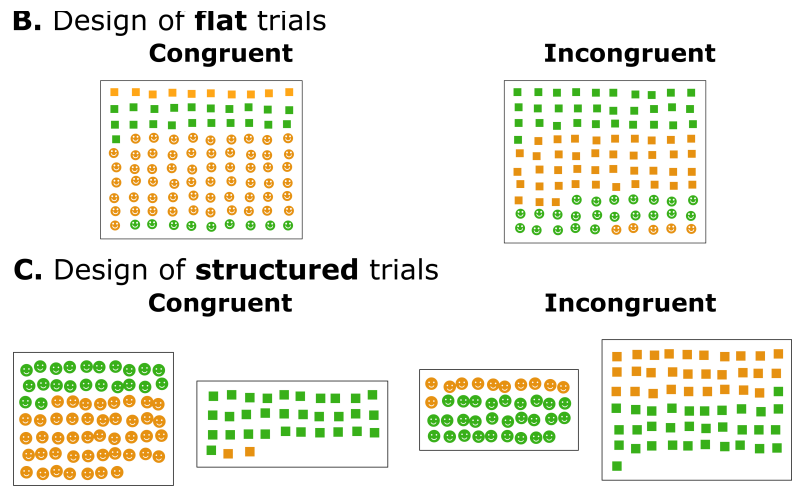

D. Design of monocolor trials Flat
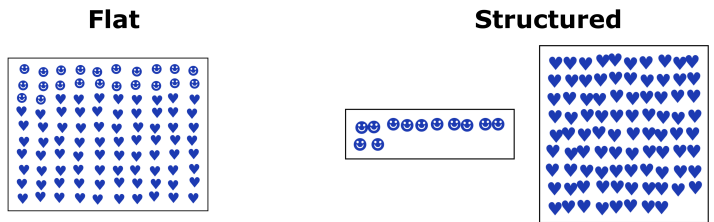

our lab. Participants were randomly assigned to either the flat or the structured condition. After the consent form, they read instructions about the task and then took 30 trials.

After the core of the experiment, we tested participants' understanding of the task. There was a crucial possible confound we controlled for: in the structured task, participants could assume that, when an element is "chosen randomly" (i) first the shape is equiprobably chosen from among the two possibilities, and (ii) then an element is sampled in the according box. This is operationally equivalent to turning the prior probabilities for each shape into .5, without changing the likelihoods. By Bayes' theorem, the posterior probabilities in such a situation align fully with the likelihoods, irreparably confounding the interpretation of participants' responses.

At the end of the experiment, first participants saw a page indicating that the instructions hadn't changed and that they shouldn't change their strategy. Then we gave participants five trials in which they could pick either of the shapes as their guess (as in the preceding trials), or guess "equally likely." Two of these trials were fillers. In the other three, all shapes had the same color but very different distributions, see Figure 1.D. Any participant who answered "equally likely" to these monocolor trials misunderstood a crucial component of the task, and was excluded from the analysis. The distributions in these monocolor trials clearly showed that one shape 
Figure 2

\section{Distribution of the trials}

(A) Global distribution of trials in our experiment, projected onto four relevant dimensions. Top-left: difference in likelihoods ( $x$ axis) vs difference in posteriors (y axis), color indicates the number of trials falling in that category. We compute the difference by subtracting the posteriors (resp. likelihood) of the non-normatively rational response from that of the normatively rational, whence the positivity of the difference in posteriors. Conversely, the difference in likelihood ranges by design from positive (congruent) to negative (incongruent) values. Top-right: distribution of the priors on the normatively rational answer. Bottom-left: distribution of the difference in posteriors. Bottom right: distribution of the difference in likelihoods. (B) Similar plot for a distribution as close as possible to the uniform distribution on both the difference in likelihood and the difference in posterior axis (see top-left; the high difference in posteriors, low difference in likelihood corner is unreachable without using more than 100 elements). Notice how while the two bottom graphs look more uniform than the distribution we used, the distribution of priors is extremely skewed toward the rational answer, thereby confounding possible strategies.
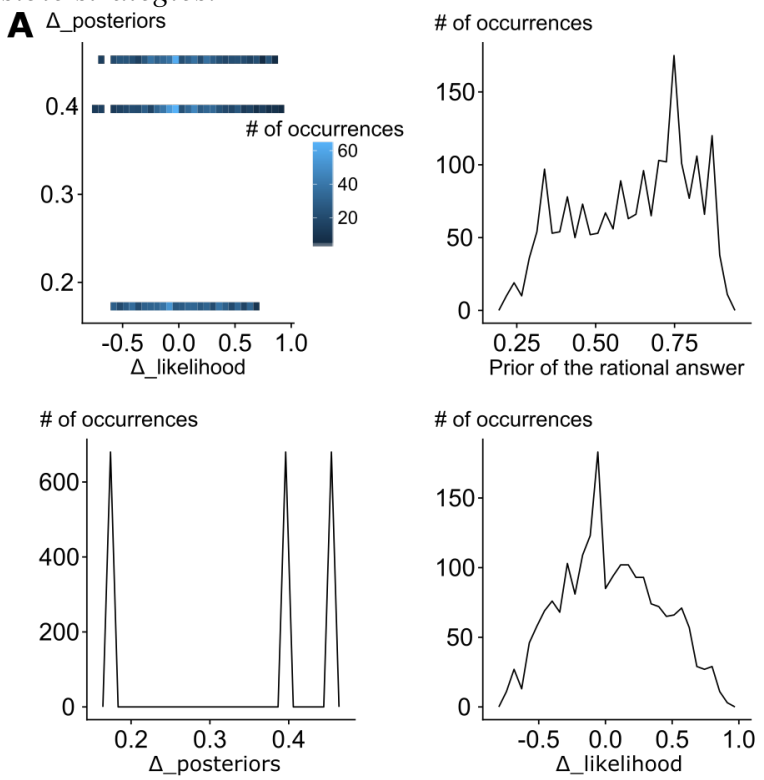

B was more likely than the other, so that the only way to answer "equally likely" was to conceive of the random selection step in a confounding way.

Additionally, we asked participants to select from a list of three statements the one they thought best described how elements were selected. We offered the following options in random order.

i. a valid description of a uniform sampling procedure,

ii. "The computer chooses an object based on which one will make the task hardest,"

iii. "A coin flip determines first which shape is selected. Then, an object is randomly selected within this shape."

Any participant who did not chose option (i) was removed from the analysis.

Materials. Participants saw 15 congruent and 15 incongruent trials in randomized order. Recall that congruent trials are those where posteriors and likelihoods point in the same direction.
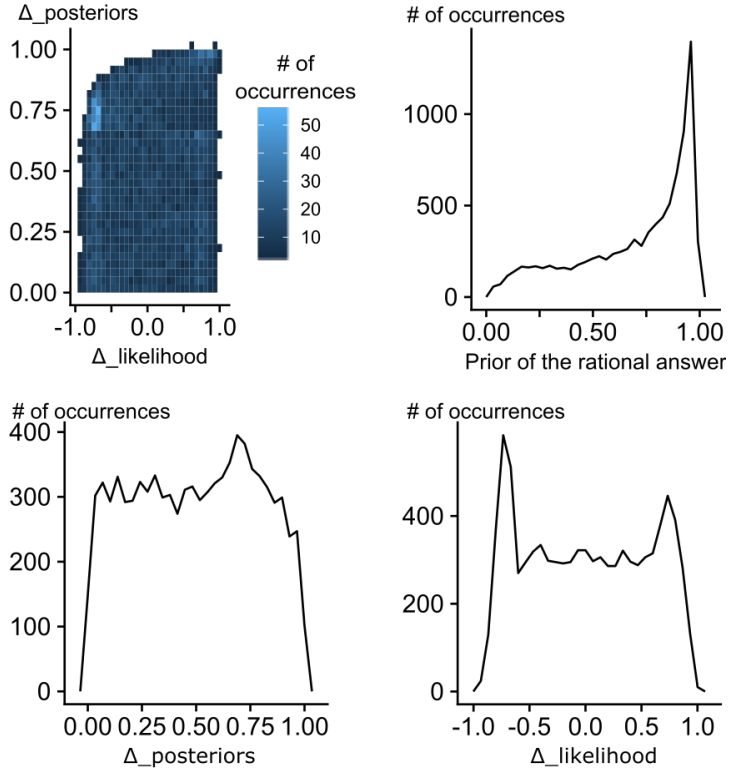

Consider the most informative formulation of Bayes' theorem from the perspective of our experimental design.

$$
P\left(h_{1} \mid e\right)=\frac{P\left(h_{1}\right) \cdot P\left(e \mid h_{1}\right)}{P\left(e \mid h_{1}\right) \cdot P\left(h_{1}\right)+P\left(e \mid h_{2}\right) \cdot\left(1-P\left(h_{1}\right)\right)}
$$

There are four terms in this equation: prior, posterior, and two likelihoods. Our theoretical hypotheses concern posteriors (rational strategy) and the two likelihood terms (confirmation-theoretic strategy). One would ideally sample trials uniformly along those axes. But of course Bayes' theorem heavily constrains this sampling space: sampling uniformly along three of the four terms will result in dramatically skewed sampling in the fourth term (see Fig. 2 for a comparison between our sampling strategy and the uniform one just described.)

The difficulty comes from the fact that it is impossible to have posteriors and likelihoods diverge markedly without posteriors aligning with priors, creating a new strategy ("follow the priors") confounded with rationality. For example, if likelihoods are strongly in favor of $h_{1}$, but posteriors are in 
favour of $h_{2}$, then (i) almost all elements of $h_{1}$ need to carry the evidence; and (ii) there need to be more elements of $h_{2}$ carrying the evidence than elements of $h_{1}$ carrying the evidence. Straightforwardly satisfying these constraints makes the prior of $h_{2}$ much greater than that of $h_{1}$. Indeed, when the prior of $h_{2}$ is much greater than that of $h_{1}$, the likelihood of $h_{1}$ increases quickly with each of its element carrying the evidence, while that of $h_{2}$ grows slowly.

We're interested in posteriors and likelihoods, but posteriors constitute the null hypothesis of rationality, so that it is more important to explore the space of likelihoods and priors. Consequently, we chose three values for posteriors, arbitrarily at .73, .7, and .58.

The visual setup of our study imposed further sampling limitations. For example, we wanted to make sure no box ever looked too empty (see Figure 1). To satisfy these constraints we settled on the following procedure. The trials were generated for each participant at the beginning of the experiment. For each trial, the procedure first decided whether the trial was congruent or not (15 of each). It then looped over one of the three possible posteriors, which determined the number of elements that needed to have the "evidence" color. Finally, it randomly picked a prior compatible with the type of trial, the posteriors and the visual constraints, which fully determines a trial. The results we obtained across participants are summarized in Fig. 2, together with a display of an example where both the difference in posteriors and the difference in likelihood are sampled uniformly.

\section{Results}

In total 20 participants answered incorrectly to the sampling check question, and 26 answered incorrectly to one of the control trials, with an overlap of 9 participants. We excluded 37 participants, ending up with a total of 68 participants in the analysis: 29 in the structured condition and 39 in the flat condition.

Participants' average rationality was high at $85 \%$ (Significant Error, $[\mathrm{SE}]=1.6 \%$ ), above what chance predicts (binomial test against chance: $p<.0001$ ).

We first ran an ANOVA on the average rationality rate per participant, with the condition (flat or structured) as a between-subjects factor and the sign of the difference in likelihood (congruency) as a within-subjects factor. This analysis is summarized in Table 1. There is no main effect of the condition, but there is a significant effect of whether a given trial was congruent or not, and a significant effect of the interaction with twice the effect size. In line with these results, Figure 3 shows that (i) overall participants are rational, (ii) the average rationality is not different between flat and structured condition, but (iii) the effect of congruency differs sharply between these two conditions.

To check the direction of the effect and collect information at the level of participants, we analyzed the distributions of within-participant differences between average congruent and incongruent trials, both on rationality rate and on response time. We ran two one-sided Wilcoxon tests and the distributions in the structured condition were significantly higher than in the flat condition for both dependent measures (rationality rate: $W=363, p=.004$; response time: $W=331, p=.002),{ }^{1}$ indicating as expected that, at the individual level, participants are slower and make more mistakes in the structured condition. The distributions can be seen in Figure 3.B.

We checked that there was no effect of the particular shapes or the color on participants' responses. Since the design matrix is deficient for these variables (each trial had to include two different symbols, and participants did not see all possible combinations of symbols and colors), we opted for a mixed-effect model with participants as random effects, and the color and shape of the rational answer as fixed effect, plus the interaction of the two. No term was significant (all $p>.5$ ), indicating that color, shape, and pair of color and shape did not significantly affect the behavior of participants in this experiment. This is has intended in our design where shapes and color were arbitrary, but we don't expect for it to hold in general - more on this in the discussion section.

To understand better what impacted the behavior of participants we ran mixed-effect models with individuals as random effects.

We first ran an intercept-only model with participants as random effects, predicting the rationality rate. The intercept was significantly different from $0(p<.0001)$ and positive, as expected given the overall rationality of the participants. This model establishes a baseline for rationality in the task independently of the exact values of $\Delta_{\text {posteriors }}$ or $\Delta_{\text {likelihoods }}$ of a given trial.

We then contrasted this model with a model with $\Delta_{\text {posteriors }}$ as fixed effects, participants as random effect and $\Delta_{\text {posteriors }}$ as random slopes, which we had to uncorrelate for the model to converge. The difference between our successive models is described in Table 2: this new model significantly differs

${ }^{1}$ For this test and the associated figure, we removed participants with a response time slower than the average plus three standard deviations, to factor out extreme outliers.

\section{Table 1}

Output of the ANOVA on average rationality as a function of condition, congruency and their interaction. $\eta_{G}^{2}$ gives a measure of effect size.

\begin{tabular}{lrrrrr}
\hline Effect & df & $\mathrm{F}$ & & $\eta_{G}^{2}$ & p.value \\
\hline Condition & 1,66 & 1.75 & & .021 & .190 \\
Congruency & 1,66 & 5.56 & $*$ & .015 & .021 \\
Interaction & 1,66 & 11.69 & $* *$ & .032 & .001 \\
\hline
\end{tabular}




\section{Figure 3}

(A) Average rationality rate in the flat and structured conditions, averaged over participants and congruency types. Error bars (in red) represent standard error of the mean per cell using the Cosineau-Morey-O'Brien method. Each point shows a participant, and as congruency was a within-subjects factor, each participant has two points on the graph. (B) Kernel density estimates of the difference, for each participant, between the Irrationality Rate (left) and Response Time (right) in the incongruent and the congruent trial types. Higher values of the difference in $x$ axis indicate less rationality (left) and longer response times (right). Color indicates the condition, flat or structured.



Condition
B

Density

Estimate

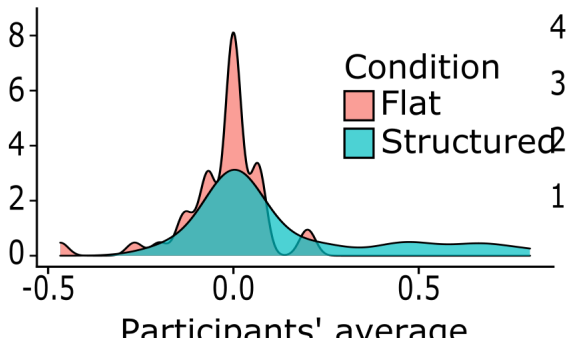

Participants' average

Irrationality Rate differences

(Incongruent - Congruent)

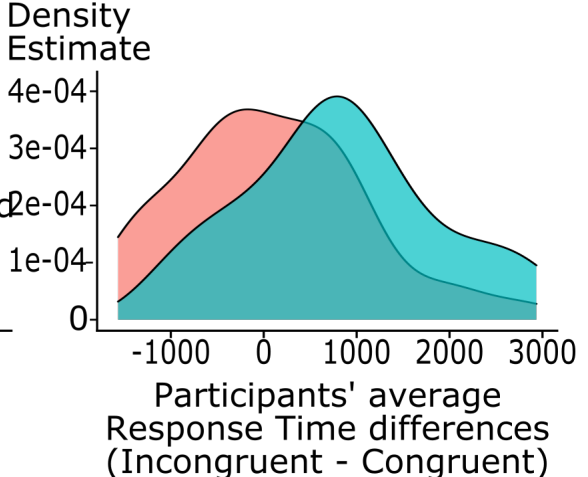

Table 2

Summary of the generalised mixed effect linear models for both dependent variables and each model structure. $\Delta_{D f}$, $\chi^{2}$, and $p$ are computed by comparing the model of that row with that of the row above. Due to convergence issues, the full model for the response time is slightly more restricted than for the rationality rate however the crucial predictor, the interaction between the difference in likelihoods and the condition, remains. The coefficients and statistics of each model are provided in supplementary materials.

\begin{tabular}{|c|c|c|c|c|c|c|c|c|c|}
\hline Dependent Variable & Model & Df & $\log (\operatorname{Lik})$ & $\Delta_{\mathrm{Df}}$ & $\chi^{2}$ & $p$ & & AIC & BIC \\
\hline \multirow{3}{*}{ Rationality Rate } & Intercept only & 2 & -646.4 & & & & & 1296.9 & 1308.1 \\
\hline & $+\Delta_{\text {Posteriors }}$ & 4 & -643.2 & 2 & 6.5 & .04 & $*$ & 1294.4 & 1316.9 \\
\hline & $+\Delta_{\text {Likelihoods }}+$ Condition: $\Delta_{\text {Likelihoods }}$ & 9 & -594.3 & 5 & 97.8 & $<.0001$ & $* * *$ & 1206.6 & 1257.2 \\
\hline \multirow{3}{*}{ Response Time } & Intercept only & 3 & -20656.7 & & & & & 41319.4 & 41336.2 \\
\hline & $+\Delta_{\text {Posteriors }}$ & 5 & -20648.2 & 2 & 17.0 & .0002 & $* * *$ & 41306.4 & 41334.5 \\
\hline & + Condition: $\Delta_{\text {Likelihoods }}$ & 7 & -20640.4 & 2 & 15.6 & .0004 & $* * *$ & 41294.7 & 41334.1 \\
\hline
\end{tabular}

from the previous one.

We take this second model as our competitor against what the theory predicts: in addition to this rationality effect, there should be an effect of $\Delta_{\text {likelihoods }}$ - and this effect should be sharper in the structured condition.

This new model has a significant term for the difference in posteriors $(p=0.014), \Delta_{\text {likelihoods }}(p=0.009)$ and the interaction ( $p=0.002$.) This model is significantly better than our baseline, with a likelihood of $-594\left(\chi^{2}=98, p<.0001\right.$, see Table 2).

We ran a similar analysis on response times. The corresponding full model did not converge: the random effect associated to the main predictor $\Delta_{\text {Likelihoods }}$ had null variance. Therefore, we removed the $\Delta_{\text {likelihoods }}$ term, keeping only its interaction with the condition (see Table 2). This model was once again significantly different from the model with only an intercept and the difference in posteriors $\left(\chi^{2}=15.6\right.$, $p=.0004)$.

Both results converge to indicate that not only does the difference in likelihoods significantly predict participants' responses and response time, but it does even more so in the structured condition.

Interestingly, when comparing the model with intercept only with the one with $\Delta$ posteriors, the difference in loglikelihood in the response time condition is bigger than the difference in the error rate condition. This suggests that the impact of $\Delta_{\text {posteriors }}$ on the decision itself is lower than its impact on the time it takes to make that decision.

\section{General discussion}

Our experiment successfully elicited "errors" in reasoning with fully explicit probability distributions. The mistakes 
occur more often as the differences in likelihood increase in favor of the normatively incorrect answer, indicating that participants rely on confirmation-theoretic measures of evidential support above and beyond the role they play in Bayes' theorem for calculating posterior probabilities. However, our design cannot distinguish between different measures of evidential support: all Bayesian confirmation-theoretic measures in the literature are possible explanations for our findings, as is a non-Bayesian, purely likelihood-based view. A more complex design, ideally employing a continuous dependent measurement, is needed to tease apart the predictions of different theories of evidential support.

Importantly, the effect is only present in the structured condition, in which the elements were clearly organized by their shapes, making the contrast between the two categories salient. This visual manipulation highlights the question element of the problem, pushing subjects to conceive of the task as strongly pitting one category against the other. This result is expected if, as we proposed, confirmation-theoretic mechanisms are triggered by a desire to address a pressing question under discussion with information meant by its utterer to help answer that question.

It is however somewhat surprising that we found no appreciable difference between congruent and incongruent trials in our flat condition. In the flat condition, all elements were presented in the same box, which plausibly makes the question-under-discussion less salient. But the task itself certainly prompted this question, by directly asking participants to choose between one of the two categories (Figure 1.A). We expected that the flat condition should show significantly less confirmation-theoretic behavior, but our theory does not explain why we found no such behavior. We conjecture that the entirely transparent probability distributions promoted posterior-like behavior so strongly that only a heavy-handed manipulation, like our structured condition, would have been enough to prompt the question-answer dynamics we propose are at the core of this phenomenon. Illuminating this issue will require systematic experimentation with multiple ways of making the question-like nature of the task salient, as well as a theory of degrees of salience of a question, which to the best of our knowledge is not currently to be found in the psychological, philosophical, or linguistic literature.

It's interesting to note that an independent study (Mangiarulo et al., 2021), looking at effects of evidential impact on perceptual probabilistic judgment, found an effect of confirmation theory in a design that shares some properties with our flat condition. The experiment by Mangiarulo et al. (2021) involved a two-step process. First, participants saw shuffled sets of about 30 elements of two possible shapes and two possible colors. These were displayed quite briefly, 3 to 4 seconds. Then the items disappeared, and participants were given the evidence on one property (either shape or color), on which to base their decision to guess the other property. They found an effect of evidential impact, that is confirmation theory, on participants' guesses and response times.

The fact that Mangiarulo et al. (2021) found an effect without structure while we didn't is informative. Besides seeing the distribution only quite briefly, their participants did not know, when looking at the distribution, what the evidence would be, or what dimension they would be asked about. This means that participants had little to no opportunity to deliberate when seeing the distribution, instead simply taking it in. This is entirely in line with the authors' intent to study effects of confirmation-theoretic measures in perception. By contrast, our design encouraged participants to explore the distribution at their leisure, and with full knowledge of the question they would be asked about it. We posit that the difference is due to the fact that our experiment is looking at higher-level cognition and deliberate decision making as intended, and not at perceptual judgment. Further work on these differing results may offer insight on the differences and similarities between deliberate decision making under complete information and perceptual judgment.

Earlier we reported an absence of effect of the shape, color, and their interaction on participants' behavior, as intended by design. However, familiar stereotypical information was a central aspect of the original explanation of the phenomenon (Kahneman \& Tversky, 1973), so that we might expect familiar stereotypes to matter when they are available to participants. In the course of designing the experiment we piloted several versions, changing the nature of controls, the sampling of the distributions, or systematically providing an "equally likely" option. The pilots differed significantly, but the same colors and symbols were used throughout, and when pooled together an intriguing, albeit statistically anecdotal curiosity emerged: in a mixed-effects model with both participants and pilot version as random effects, and shapecolor interaction as fixed effect, there was an overall effect of the interaction (Linear mixed model, t-tests use Satterthwaite's method $F_{20,10854.72}=1.73, p=.023$ ), entirely driven by two colors for the heart shape: red, making participants more prone to confirmation-theoretic behavior $(t=-2.06$, $p=.0395)$ and green making participants less prone to it $(t=2.33, p=.0198)$. This hints at a substantive role for familiar stereotypes in the broader phenomenon. Our experiment shows that familiar stereotypes are by no means a necessary condition for confirmation-theoretic responses, but we found suggestive evidence that their presence might potentiate such behavior.

\section{Funding}

The research presented here was supported by Agence Nationale de la Recherche grants ANR-17-EURE-0017 (FrontCog, Department of Cognitive Studies, Ecole Normale Supérieure) and ANR-18-CE28-0008 (LANG-REASON; PI: Mascarenhas). 


\section{Data availability statement}

All the data and analysis scripts for this article are shared on the publicly available OSF repository https://osf.io/ qp5mz/?view_only=97d4ee656ca04e74accb15d645365c $2 \mathrm{a}$

\section{References}

Alonso-Ovalle, L. (2006). Disjunction in alternative semantics (PhD diss.). UMass Amherst.

Crupi, V., Fitelson, B., \& Tentori, K. (2008). Probability, confirmation, and the conjunction fallacy. Thinking $\mathcal{E}$ Reasoning, 14(2), 182-199.

De Leeuw, J. R. (2015). Jspsych: A javascript library for creating behavioral experiments in a web browser. Behavior research methods, 47(1), 1-12.

Frank, M. C., \& Goodman, N. D. (2012). Predicting pragmatic reasoning in language games. Science, 336(6084), 998-998.

Gigerenzer, G., \& Hoffrage, U. (1995). How to improve Bayesian reasoning without instruction: Frequency formats. Psychological Review, 102(4), 684-704.

Goodman, N. D., \& Frank, M. C. (2016). Pragmatic language interpretation as probabilistic inference. Trends in cognitive sciences, 20(11), 818-829.

Groenendijk, J. (2008). Inquisitive Semantics: Two possibilities for disjunction. Proceedings of the Seventh International Tbilisi Symposium on Language, Logic and Computation.

Jayaseelan, K. (2001). Questions and question-word incorporating quantifiers in Malayalam. Syntax, 4(2), 6393.

Jayaseelan, K. (2008). Question particles and disjunction [Unpublished manuscript: The English and Foreign Languages University (Hyderabad)]. http://ling.auf. net/lingBuzz/000644/current.pdf

Kahneman, D., \& Tversky, A. (1973). On the psychology of prediction. Psychological Review, 80(4), 237-251.

Koralus, P., \& Mascarenhas, S. (2013). The erotetic theory of reasoning: Bridges between formal semantics and the psychology of deductive inference. Philosophical Perspectives, 27, 312-365.

Kratzer, A., \& Shimoyama, J. (2002). Indeterminate pronouns: The view from Japanese. Third Tokyo Conference on Psycholinguistics.

Mangiarulo, M., Pighin, S., Polonio, L., \& Tentori, K. (2021). The effect of evidential impact on perceptual probabilistic judgments. Cognitive Science, 45(1), e12919.

Mascarenhas, S. (2009). Inquisitive semantics and logic (Master's thesis). ILLC.

Oaksford, M., \& Chater, N. (2007). Bayesian rationality: The probabilistic approach to human reasoning. Oxford University Press.

Sablé-Meyer, M., \& Mascarenhas, S. (2021). Indirect illusory inferences from disjunction: A new bridge between deductive inference and representativeness. Review of Philosophy and Psychology, 12(2). https: //doi.org/10.1007/s13164-021-00543-8

Schwarz, N., Strack, F., Hilton, D. J., \& Naderer, G. (1991). Base rates, representativeness, and the logic of conversation: The contextual relevance of "irrelevant" information. Social Cognition, 9(1), 67-84.

Szabolcsi, A., Whang, J. D., \& Zu, V. (2014). Quantifier words and their multi-functional parts. Language and Linguistics, 15(1), 115-155.

Tenenbaum, J. B., \& Griffiths, T. L. (2001). The rational basis of representativeness. In J. D. Moore \& K. Stenning (Eds.), Proceedings of the 23rd annual conference of the cognitive science society (pp. 1036-1042).

Tentori, K., Crupi, V., \& Russo, S. (2013). On the determinants of the conjunction fallacy: Probability versus inductive confirmation. Journal of Experimental Psychology: General, 142(1), 235-255.

Tversky, A., \& Kahneman, D. (1983). Extensional versus intuitive reasoning: The conjunction fallacy in probability judgment. Psychological Review, 90, 293315. 